\title{
Lusioersily
}

\section{New results in disturbance decoupled fault reconstruction in linear uncertain systems using two sliding mode observers in cascade}

Ng, K., Tan, C. P., Man, Z., \& Akmeliawati, R. (Accepted/ln press). New results in disturbance decoupled fault reconstruction in linear uncertain systems using two sliding mode observers in cascade. International Journal of Control, Automation and Systems, 8(3), 506-518. https://doi.org/10.1007/s12555-010-0303-8

Link to publication record in Ulster University Research Portal

\section{Published in:}

International Journal of Control, Automation and Systems

\section{Publication Status:}

Accepted/In press: 04/12/2009

DOI:

10.1007/s12555-010-0303-8

\section{Document Version}

Publisher's PDF, also known as Version of record

\section{General rights}

Copyright for the publications made accessible via Ulster University's Research Portal is retained by the author(s) and / or other copyright owners and it is a condition of accessing these publications that users recognise and abide by the legal requirements associated with these rights.

\section{Take down policy}

The Research Portal is Ulster University's institutional repository that provides access to Ulster's research outputs. Every effort has been made to ensure that content in the Research Portal does not infringe any person's rights, or applicable UK laws. If you discover content in the Research Portal that you believe breaches copyright or violates any law, please contact pure-support@ulster.ac.uk. 


\title{
New Results in Disturbance Decoupled Fault Reconstruction in Linear Uncertain Systems Using Two Sliding Mode Observers in Cascade
}

\author{
Kok Yew Ng, Chee Pin Tan*, Zhihong Man, and Rini Akmeliawati
}

\begin{abstract}
This paper presents a disturbance decoupled fault reconstruction (DDFR) scheme using two sliding mode observers in cascade. Measurable signals from the first observer are found to be the output of a fictitious system that is driven by the fault and disturbances. Then the signals are fed into a second observer which will reconstruct the fault. Sufficient conditions which guarantee DDFR are investigated and presented in terms of the original system matrices, and they are found to be less conservative than if only one single observer is used; therefore DDFR can be achieved for a wider class of systems using two sliding mode observers. A simulation example validates the claims made in this paper.
\end{abstract}

Keywords: Disturbance decoupling, fault reconstruction, robustness, sliding mode observer.

\section{INTRODUCTION}

Fault detection and isolation (FDI) is an important area of research activity. A fault is deemed to occur when the system being monitored is subject to an abnormal condition [2]. The fundamental purpose of an FDI scheme is to generate an alarm when a fault occurs (detection) and also to identify the nature and location of the fault (isolation). A special class of problem within the field of FDI is the problem of fault reconstruction $[4,5,13]$, which not only detects and isolates, but provides an estimate of the fault so that its shape and magnitude can be better understood and more precise corrective action can be taken. However, most fault reconstruction schemes are designed about a model, which usually does not perfectly represent the system as certain dynamics are either unknown or do not fit exactly into the framework of the model. These dynamics are usually represented as a class of disturbances within the model [11] and could corrupt the reconstruction; producing a nonzero reconstruction when there are no faults, or worse, mask the effect of a fault. Therefore, schemes need to be designed so that the reconstruction is robust to disturbances.

Edwards et al. [4,5] used a sliding mode observer [3] to reconstruct faults, in which there was no explicit

Manuscript received June 7, 2008; revised July 26, 2009; accepted December 4, 2009. Recommended by Editorial Board member Duk-Sun Shim under the direction of Editor Jae Weon Choi.

Kok Yew Ng and Chee Pin Tan are with the School of Engineering, Monash University, Jalan Lagoon Selatan, 46150 Malaysia (e-mails: \{ng.kok.yew, tan.chee.pin\}@eng.monash.edu.my).

Zhihong Man is with the Robotics and Mechatronics Engineering, Swinburne University of Technology, PO Box 218, Hawthorn, Victoria, 3122 Australia (e-mail: zman@swin.edu.au).

Rini Akmeliawati is with the Kuliyyah of Engineering, International Islamic University, Jalan Gombak 53100 Kuala Lumpur, Malaysia (e-mail: rakmelia@iiu.edu.my). consideration of the disturbances or uncertainty. Tan \& Edwards [15] built on the work in [4,5] and presented a design algorithm for the observer, using Linear Matrix Inequalities (LMIs) [1], such that the $\mathscr{L}_{2}$ gain from the disturbances to the fault reconstruction is minimized. Saif \& Guan [13] aggregated the faults and disturbances to form a new 'fault' vector and used a linear unknown input observer to reconstruct the new 'fault' vector. Although this successfully decouples the disturbances from the fault reconstruction, it requires very stringent conditions to be fulfilled, and is conservative because the disturbance does not need to be reconstructed, only rejected/decoupled. Edwards \& Tan later [6] compared the fault reconstruction performances of [5] and [13], and found that it was not necessary to reconstruct the disturbance in order to generate a disturbance decoupled fault reconstruction (DDFR). A counter example was presented in [6] to demonstrate this, but the conditions for disturbance decoupling were not formally investigated. $\mathrm{Ng}$ et al. [8,9] built on the work of [6] and analyzed theconditions that guarantee DDFR using the sliding mode observer [3]. It was also found in [8,9] that the sliding mode observer can achieve DDFR with weaker conditions compared with the linear observer.

This paper further builds on the work in $[8,9]$ by using two sliding mode observers in cascade, where measurable signals from the first observer are found to be the output of a fictitious system that is driven by the faults and disturbance, and fed into a second sliding mode observer. The second observer then reconstructs the fault. The conditions that guarantee DDFR are then investigated, and it was found that the conditions are less conservative than those found in $[8,9]$, which meant that the scheme proposed in this paper are applicable to a wider class of systems compared to if only one observer was used [8,9]. In addition, the sufficient conditions arefound to be easily testable in terms of the original system matrices, which means that the user can know 
immediately from the outset whether the scheme proposed in this paper can achieve DDFR or not.

This paper is organized as follows: Section 2 outlines the problem statement, presents previous work as the basis of the work in this paper and states the main result; Section 3 presents the 2-observer DDFR scheme in this paper; Section 4 investigates thesufficient conditions in terms of the original system matrices; Section 5 presents a numerical example to validate the scheme and Section 6 concludes the paper.

\section{PRELIMENARIES AND PROBLEM STATEMENT}

Consider the following system

$$
\begin{aligned}
& \dot{x}=A x+M f+Q \xi, \\
& y=C x,
\end{aligned}
$$

where $x \in \mathbb{R}^{n}$ are the states, $y \in \mathbb{R}^{p}$ are the outputs and $f \in \mathbb{R}^{q}$ are unknown faults. The signals $\xi \in \mathbb{R}^{h}$ are uncertainties or dynamics that represent the mismatch between the linear model (1)-(2) and the real plant. Without loss of generality assume that $\operatorname{rank}(M)=q$, $\operatorname{rank}(C)=p$ and $\operatorname{rank}(C Q)=k_{1}<h$. Assume also the following

N1. $\operatorname{rank}(C M)=\operatorname{rank}(M)$,

$\mathrm{N} 2 . \operatorname{rank}\left(C\left[\begin{array}{ll}M & Q\end{array}\right]\right)=\operatorname{rank}(C M)+\operatorname{rank}(C Q)$.

The objective is to generate a reconstruction of $f$ that is not affected by $\xi$.

Proposition 1: If Assumptions N1 - N2 hold, then there exists a change of coordinates $x \mapsto T_{1} x, \quad \xi \mapsto$ $T_{\xi}^{-1} \xi$ such that the matrices $(A, M, Q, C)$ have the structure

$$
\begin{aligned}
& A=\left[\begin{array}{ll}
\frac{n-p}{A_{1}} & \frac{p}{A_{2}} \\
A_{3} & A_{4}
\end{array}\right] \begin{array}{l}
\uparrow n-p \\
\uparrow p
\end{array} \quad M=\left[\begin{array}{c}
\frac{q}{0} \\
M_{2}
\end{array}\right] \begin{array}{l}
\uparrow n-p, \\
\uparrow p,
\end{array}
\end{aligned}
$$

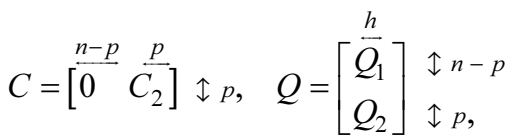

where $M_{2}$ can be further partitioned to be

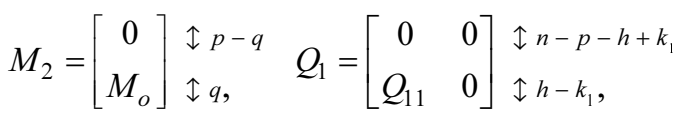

$$
\begin{aligned}
& Q_{2}=\left[\begin{array}{cc}
0 & 0 \\
0 & Q_{22} \\
0 & 0
\end{array}\right] \begin{array}{l}
\mathfrak{\downarrow} p-q-k_{1} \\
\mathfrak{} k_{1},
\end{array}
\end{aligned}
$$

where $C_{2}, M_{o}, Q_{11}$ and $Q_{22}$ are square and invertible.

Proof: The proof is taken from [8,9] and is available in Appendix A.

In the coordinates of (3)-(4), further partition $A$ to be

$$
\left[\begin{array}{l|l}
A_{1} & \star \\
\hline A_{31} & \star \\
A_{32} & \star \\
A_{33} & \star
\end{array}\right] \begin{aligned}
& \mathfrak{i}-p \\
& \uparrow k_{1} \\
& \uparrow q,
\end{aligned}
$$

where $\star$ are matrices with $p$ columns that play no role in the following analysis. Then further partition $A$ in (7) as

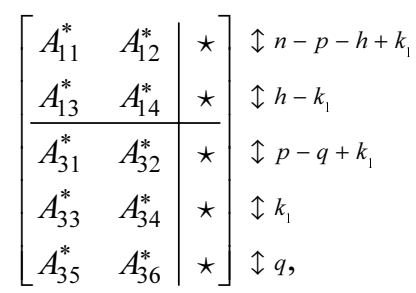

where $A_{11}^{*}, A_{14}^{*}$ are square matrices.

In $[8,9]$, it is possible to generate a reconstruction of $f$ that is independent of $\xi$ (and achieve DDFR) using a sliding mode observer [3] if the following conditions are satisfied

A1. $\left(A,\left[\begin{array}{ll}M & Q\end{array}\right], C\right)$ is minimum phase

A2. $\operatorname{rank}\left(A_{32}^{*}\right)=\operatorname{rank}\left[\begin{array}{c}A_{12}^{*} \\ A_{32}^{*} \\ A_{36}^{*}\end{array}\right]$.

2.1. Previous work

A sliding mode observer [3] for the system (1)-(2) of the form

$$
\begin{aligned}
& \dot{\hat{x}}=A \hat{x}-G_{l} e_{y}+G_{n} v, \\
& \hat{y}=C \hat{x},
\end{aligned}
$$

where $\hat{x} \in \mathbb{R}^{n}$ is the estimate of the state $x$ and $e_{y}=\hat{y}-y$ is the output estimation error. The term $v$ is a nonlinear discontinuous term defined by

$$
v=-\rho \frac{e_{y}}{\left\|e_{y}\right\|} \quad \text { if } e_{y} \neq 0
$$

where the positive scalar function $\rho$ is an upper bound of $f$ and $\xi$. The matrices $G_{l}, G_{n} \in \mathbb{R}^{n \times p}$ are the observer gains to be designed. In the coordinates of (3) (14), $G_{n}$ is assumed to have the structure

$$
G_{n}=\left[\begin{array}{c}
-L \\
I_{p}
\end{array}\right]\left(P_{o} C_{2}\right)^{-1}, L=\left[\begin{array}{ll}
L_{1} & 0
\end{array}\right],
$$

where $P_{o} \in \mathbb{R}^{p \times p}$ is a symmetric positive definite (s.p.d.) matrix and $L_{1} \in \mathbb{R}^{(n-p) \times(p-q)}$.

Define the state estimation error as $e:=\hat{x}-x$. Combining (1)-(2) and (9)-(10), results in

$$
\dot{e}=\left(A-G_{l} C\right) e+G_{n} v-M f-Q \xi .
$$


Lemma 1 [15]: If there exists a value of $G_{l}$ that satisfies $P\left(A-G_{l} C\right)+\left(A-G_{l} C\right)^{T} P<0$ where

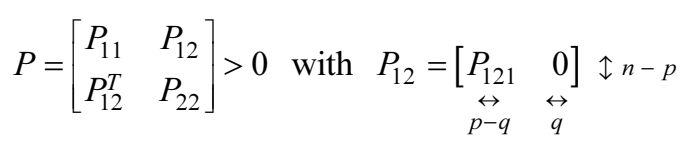

then if $P_{o}=P_{22}-P_{12}^{T} P_{11}^{-1} P_{12}$, and for a large enough choice of $\rho$, an ideal sliding motion takes place on $\mathscr{S}=\{e: C e=0\}$ in finite time.

Apply a change of coordinates such that $e_{L}:=\operatorname{col}\left(e_{1}\right.$, $\left.e_{y}\right)=T_{L} e$ where

$$
T_{L}:=\left[\begin{array}{cc}
I_{n-p} & L \\
0 & C_{2}
\end{array}\right] .
$$

Then assume a sliding motion has taken place at $\mathscr{S}$, and therefore (13) in the new coordinates can be partitioned to be (see Section 2.2 of [15])

$$
\begin{aligned}
& \dot{e}_{1}=\left(A_{1}+L A_{3}\right) e_{1}-\left(Q_{1}+L Q_{2}\right) \xi, \\
& 0=C_{2} A_{3} e_{1}+P_{o}^{-1} v_{e q}-C_{2} M_{2} f-C_{2} Q_{2} \xi,
\end{aligned}
$$

where $v_{e q}$ is the equivalent output error injection term required to maintain the sliding motion [5] and can be approximated to any degree of accuracy [5] by replacing $v$ with

$$
v=-\rho \frac{e_{y}}{\left\|e_{y}\right\|+\delta},
$$

where $\delta$ is a small positive scalar. Since $e_{y}$ is measurable, $v_{e q}$ can be computed online. See [5] for full details.

In $[8,9]$, a fault reconstruction $\hat{f}$ was defined as $\hat{f}:=W C_{2}^{-1} P_{o}^{-1} v_{e q}, \quad W:=\left[\begin{array}{lll}W_{1} & 0 & M_{o}^{-1}\end{array}\right]$ where $W_{1} \in$ $\mathbb{R}^{q \times\left(p-q-k_{1}\right)}$ is design freedom. Define $v:=-e_{1}$, $e_{f}:=\hat{f}-f$, pre-multiply (16) with $W C_{2}^{-1}$ and rearrange (15)-(16) to obtain the pair of equations

$$
\begin{aligned}
& \dot{v}=\mathscr{A} v+\mathscr{B} \xi, \\
& e_{f}=\mathscr{C} v,
\end{aligned}
$$

where

$$
\mathscr{A}:=A_{1}+L A_{3}^{*} \equiv\left[\begin{array}{ll}
\mathscr{A}_{1} & \mathscr{L}_{2} \\
\mathscr{2} / 3 & \mathscr{L}_{4}
\end{array}\right],
$$

$$
\begin{aligned}
\mathscr{B} & :=Q_{1}+L Q_{2}=\left[\begin{array}{cc}
0 & L_{12} Q_{22} \\
Q_{11} & L_{14} Q_{22}
\end{array}\right] \equiv\left[\begin{array}{cc}
0 & \mathscr{C}_{2} \\
\mathscr{S}_{3} & \mathscr{F}_{4}
\end{array}\right], \\
\mathscr{C} & :=\left[\begin{array}{ll}
W_{1} A_{31}^{*}+M_{o}^{-1} A_{35}^{*} & W_{1} A_{32}^{*}+M_{o}^{-1} A_{36}^{*}
\end{array}\right] \\
& \equiv\left[\begin{array}{ll}
\mathscr{C}_{1} & C_{2}
\end{array}\right],
\end{aligned}
$$

where $\mathscr{L}_{1}=A_{11}^{*}+L_{11} A_{31}^{*}+L_{12} A_{33}^{*}, \quad \mathscr{L}_{2}=A_{12}^{*}+L_{11} A_{32}^{*}+$ $L_{12} A_{34}^{*}, \mathscr{A}_{3}=A_{13}^{*}+L_{13} A_{31}^{*}+L_{14} A_{33}^{*}$ and $\mathscr{A}_{4}=A_{14}^{*}+L_{13} A_{32}^{*}$ $+L_{14} A_{34}^{*}$.

If the system (18)-(19) is made zero, then $e_{f} \equiv 0$ and disturbance decoupling fault reconstruction is achieved.

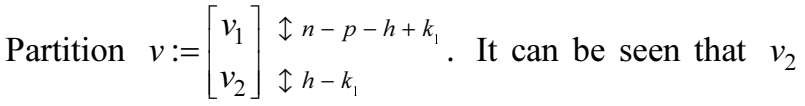
will always be affected by $\xi$ because $\mathscr{J}_{3}$ is full rank. However, $v_{1}$ can be decoupled from $\xi$ if $\mathscr{z}_{2}$ and $\mathscr{L}_{12}=0$; this would require $L_{12}=0$ and $A_{12}^{*}+L_{11} A_{32}^{*}$ $=0$, which in turn requires $\operatorname{rank}\left(A_{32}^{*}\right)=\operatorname{rank}\left[\begin{array}{l}A_{12}^{*} \\ A_{32}^{*}\end{array}\right]$. Then $e_{f}$ can be decoupled from $v_{2}$ (and therefore from $\xi)$ if $\mathscr{C}_{2}=0$ which requires $W_{1} A_{32}^{*}+M_{o}^{-1} A_{36}^{*}=0$ which in turn requires $\operatorname{rank}\left(A_{32}^{*}\right)=\operatorname{rank}\left[\begin{array}{c}A_{36}^{*} \\ A_{32}^{*}\end{array}\right]$. Combining the rank requirements results in Condition A2. Then Condition A1 guarantees that the remaining degrees of freedom in $L$ can be chosen such that $\mathscr{A}$ is stable.

\subsection{Main result}

This paper proposes a scheme to achieve DDFR when Condition A2 is not satisfied. The main result of this paper is summarized in the following theorem:

Theorem 1: DDFR can be achieved using a 2observer structure in Figure 1 if the following conditions are satisfied

B1. $\left(A,\left[\begin{array}{ll}M & Q\end{array}\right], C\right)$ is minimum phase

B2. $\operatorname{rank}\left(X_{1}\right)-\operatorname{rank}\left(X_{2}\right)=\operatorname{rank}(Q)$ where

$$
\begin{aligned}
X_{1} & =\left[\begin{array}{cccccc}
A Q & 0 & 0 & Q & 0 & 0 \\
C Q & 0 & 0 & 0 & 0 & 0 \\
C A Q & C Q & 0 & 0 & C M & 0 \\
C A^{2} Q & C A Q & C Q & 0 & C A M & C M
\end{array}\right], \\
X_{2} & =\left[\begin{array}{ccccc}
C Q & 0 & 0 & 0 & 0 \\
C A Q & C Q & 0 & C M & 0 \\
C A^{2} Q & C A Q & C Q & C A M & C M
\end{array}\right] .
\end{aligned}
$$

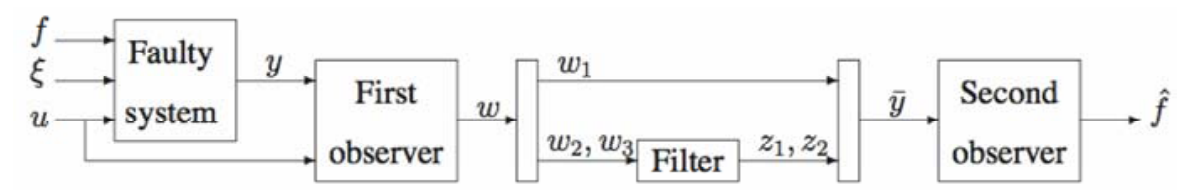

Fig. 1. Schematic diagram of the scheme proposed in this paper. 
The remainder of this paper provides a constructive proof of Theorem 1 .

\section{DDFR USING TWO OBSERVERS}

For ease of analysis, a coordinate transformation is introduced as follows in the sequel. Define $k_{2}:=$ rank $\left(A_{32}^{*}\right)$ and $p_{2}:=\operatorname{rank}\left[\begin{array}{ll}A_{31}^{*} & A_{32}^{*}\end{array}\right]+q+k_{1}$ where $p_{2} \leq$ $p$. Then let $R_{1} \in \mathbb{R}^{\left(p-q-k_{1}\right) \times\left(p-q-k_{1}\right)}$ be an orthogonal matrix such that

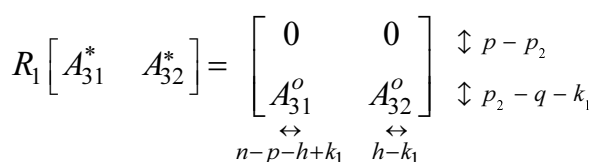

and $\quad R_{2} \in \mathbb{R}^{\left(p_{2}-q-k_{1}\right) \times\left(p_{2}-q-k_{1}\right)} \quad$ and $\quad R_{3} \in \mathbb{R}^{\left(h-k_{1}\right) \times\left(h-k_{1}\right)}$ to be orthogonal matrices such that

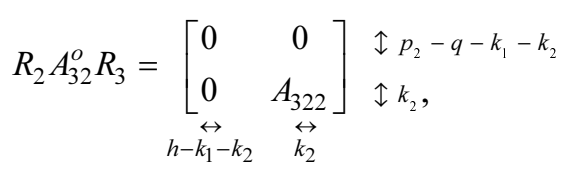

where $A_{322}$ has full rank, and assume the following general partitions:

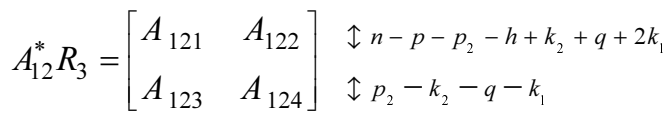

$$
\begin{aligned}
& A_{36}^{*} R_{3}=\left[\begin{array}{ll}
A_{361} & A_{362}
\end{array}\right] \uparrow q . \\
& \overleftrightarrow{k_{2}}
\end{aligned}
$$

If Condition $\mathrm{A} 2$ is satisfied, then $A_{121}, A_{123}$ and/or $A_{361}$ will all be zero. However, in this paper, no such constraint is in place and $A_{121}, A_{123}$ and/or $A_{361}$ are general matrices.

Then let $R_{4} \in \mathbb{R}^{\left(n-p-h+k_{1}\right) \times\left(n-p-h+k_{1}\right)}$ be an orthogonal matrix such that

$$
\begin{aligned}
& R_{2} A_{31}^{o} R_{4}=\left[\begin{array}{cc}
0 & A_{3112} \\
A_{3121} & A_{3112}
\end{array}\right] \begin{array}{l}
\mathfrak{\imath} p_{2}-q-k_{1}-k_{2} \\
\uparrow k_{2},
\end{array} \\
& n-p-p_{2}-\stackrel{\leftrightarrow}{h+k_{2}}+q+2 k_{1} \quad \stackrel{\leftrightarrow}{p_{2}-q-k_{1}-k_{2}}
\end{aligned}
$$

where $A_{3112}$ is full rank. It is straightforward to show that

$$
\begin{aligned}
& {\left[\begin{array}{cc}
I_{p-p_{2}} & 0 \\
0 & R_{2}
\end{array}\right] R_{1}\left[\begin{array}{ll}
A_{31}^{*} & A_{32}^{*}
\end{array}\right]\left[\begin{array}{cc}
R_{4} & 0 \\
0 & R_{3}
\end{array}\right]} \\
& =\left[\begin{array}{cccc}
0 & 0 & 0 & 0 \\
0 & A_{3112} & 0 & 0 \\
A_{3121} & A_{3122} & 0 & A_{322}
\end{array}\right] .
\end{aligned}
$$

Define a coordinate transformation $Z:=Z_{1} Z_{2} Z_{3}$ where

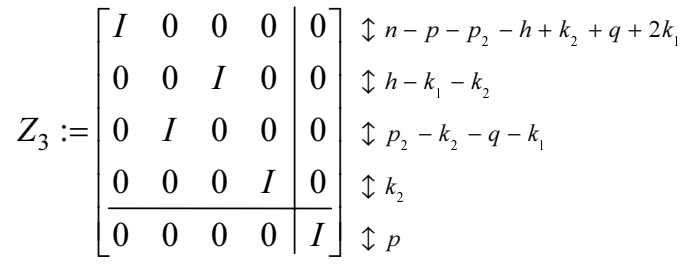

$$
\begin{aligned}
& Z_{2}:=\left[\begin{array}{ccc|c}
I_{n-p-p_{2}-h+k_{2}+q+2 k_{1}} & 0 & 0 & 0 \\
0 & I_{p_{2}-2 k_{2}-q-2 k_{1}+h} & 0 & 0 \\
A_{3121} A_{322}^{-1} & 0 & I_{k_{2}} & 0 \\
\hline 0 & 0 & 0 & I_{p}
\end{array}\right] \\
& Z_{3}:=\left[\begin{array}{cc|ccc}
R_{4}^{-1} & 0 & 0 & 0 \\
0 & R_{3}^{-1} & 0 & 0 \\
\hline 0 & 0 & {\left[\begin{array}{cc}
I_{p-p_{2}} & 0 \\
0 & R_{2}
\end{array}\right] R_{1}} & 0 \\
0 & 0 & 0 & I_{q+k_{1}}
\end{array}\right]
\end{aligned}
$$

in order to obtain

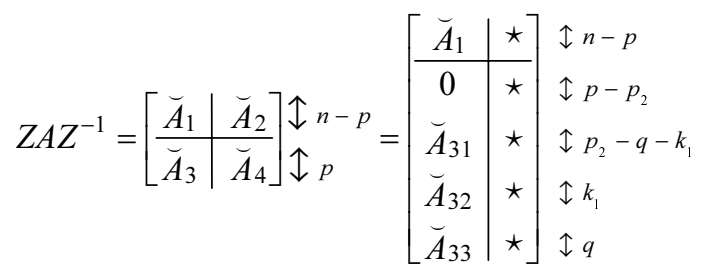

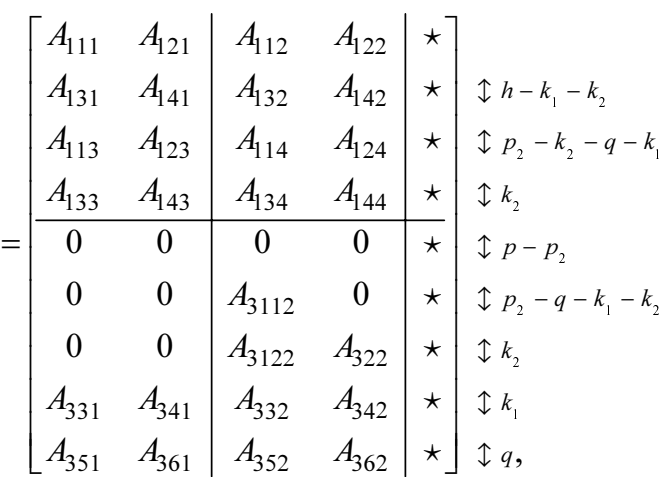

where $\star$ are matrices with $p$ columns and play no role in the following analysis. It is clear that $\breve{A}_{31}=\left[\begin{array}{ll}0 & \breve{A}_{312}\end{array}\right]$ where $\breve{A}_{312}:=\left[\begin{array}{cc}A_{3112} & 0 \\ A_{3122} & A_{322}\end{array}\right]$ is square and invertible. In addition, $Q$ and $M$ are transformed to be

$$
Z Q=\left[\begin{array}{c}
\breve{Q}_{1} \\
\breve{Q}_{2}
\end{array}\right]=\left[\begin{array}{cc}
0 & 0 \\
Q_{111} & 0 \\
0 & 0 \\
Q_{112} & 0 \\
\hline 0 & 0 \\
0 & 0 \\
0 & 0 \\
0 & Q_{22} \\
0 & 0
\end{array}\right],
$$




$$
\begin{aligned}
& Z M=\left[\begin{array}{c}
0 \\
\breve{M}_{2}
\end{array}\right]=\left[\begin{array}{c}
0 \\
0 \\
0 \\
0 \\
0 \\
0 \\
0 \\
0 \\
M_{o}
\end{array}\right], \\
& C Z^{-1}=\left[\begin{array}{ll}
0 & \breve{C}_{2}
\end{array}\right],
\end{aligned}
$$

where

$$
\begin{aligned}
& {\left[\begin{array}{l}
Q_{111} \\
Q_{112}
\end{array}\right]=R_{3}^{-1} Q_{11},}
\end{aligned}
$$

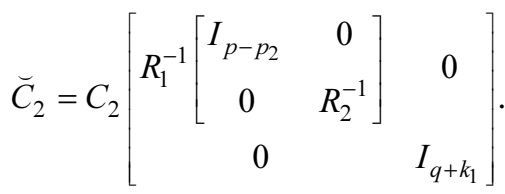

\subsection{The system for the second observer}

Implement the first sliding mode observer as described in Section 2.1, except that the matrix $L_{1}$ in $L$ from (12) has a different dimension as follows

$$
L \in \mathbb{R}^{(n-p) \times p}=\left[\begin{array}{ll}
L_{1} & 0
\end{array}\right], L_{1} \in \mathbb{R}^{(n-p) \times\left(p-q-k_{1}\right)} .
$$

Choose a matrix $L_{1}$ such that $\breve{A}_{1}+L_{1} \breve{A}_{31}$ is stable. Define $v:=-e_{1}$ and $w:=\left(P_{o} \breve{C}_{2}\right)^{-1} v_{e q}$ and re-arrange (15) - (16) to respectively obtain

$$
\begin{aligned}
& \dot{v}=\left(\breve{A}_{1}+L \breve{A}_{3}\right) v+\left(\breve{Q}_{1}+L \breve{Q}_{2}\right) \xi, \\
& w=\breve{A}_{3} v+\breve{Q}_{2} \xi+\breve{M}_{2} f .
\end{aligned}
$$

From the structures of $\breve{A}_{3}, \breve{Q}_{2}$ and $\breve{M}_{2}$ from (28)(30), it is clear that the top $p-p_{2}$ components of $w$. Hence $w$ can be partitioned as follows

$$
w=\left[\begin{array}{r}
0 \\
w_{1} \\
w_{2} \\
w_{3}
\end{array}\right] \mathfrak{l} p-p_{2}-k_{2} .
$$

Define $\breve{Q}_{22}:=\left[\begin{array}{ll}0_{k_{2} \times\left(h-k_{1}\right)} & Q_{22}\end{array}\right]$ and substituting for $\breve{A}_{3}, \breve{Q}_{2}$ and $\breve{M}_{2}$ from (28)-(30) into (36), then $w_{1}-w_{3}$ in (37) can be expanded to be

$$
\begin{aligned}
& w_{1}=\breve{A}_{31} v, \\
& w_{2}=\breve{A}_{32} v+\breve{Q}_{22} \xi, \\
& w_{3}=\breve{A}_{33} v+M_{o} f .
\end{aligned}
$$

Then define $\mathrm{z}_{1}, \mathrm{z}_{2}$ to be filtered versions of $w_{2}, w_{3}$ representing

$$
\dot{z}_{1}=-\alpha_{1} z_{1}+\alpha_{1} w_{2}, \dot{z}_{2}=-\alpha_{2} z_{2}+\alpha_{2} w_{3},
$$

where $\alpha_{1}, \alpha_{2} \in \mathbb{R}_{+}$. Substituting from (39)-(40) into (41) to get the following analytical expressions for $z_{1}$ and $z_{2}$ :

$$
\begin{aligned}
& z_{1}=-\alpha_{1} z_{1}+\alpha_{1} \breve{A}_{32} v+\alpha_{1} \breve{Q}_{22} \xi, \\
& z_{2}=-\alpha_{2} z_{2}+\alpha_{2} \breve{A}_{33} v+\alpha_{2} M_{o} f .
\end{aligned}
$$

Then equations (35), (38), (42) and (43) can be combined to form another system of order $n_{2}:=n-p$ $+q+k_{1}$ with a measurable output of dimension $p_{2}$

$$
\begin{aligned}
& \dot{\bar{x}}=\bar{A} \bar{x}+\bar{Q} \xi+\bar{M} f, \\
& \bar{y}=\bar{C} \bar{x},
\end{aligned}
$$

where $\bar{x}:=\left[\begin{array}{c}v \\ z_{1} \\ z_{2}\end{array}\right], \bar{y}:=\left[\begin{array}{c}w_{1} \\ z_{1} \\ z_{2}\end{array}\right]$ and

$$
\begin{aligned}
& \bar{A}=\left[\begin{array}{ccc}
\breve{A}_{1}+L \breve{A}_{3} & 0 & 0 \\
\alpha_{1} \breve{A}_{32} & -\alpha_{1} I_{k_{1}} & 0 \\
\alpha_{2} \breve{A}_{33} & 0 & -\alpha_{2} I_{q}
\end{array}\right], \bar{Q}=\left[\begin{array}{c}
\breve{Q}_{1} \\
\breve{Q}_{22} \\
0
\end{array}\right], \\
& \bar{M}=\left[\begin{array}{c}
0 \\
0 \\
M_{o}
\end{array}\right], \quad \bar{C}=\left[\begin{array}{ccc}
\breve{A}_{1} & 0 & 0 \\
0 & I_{k_{1}} & 0 \\
0 & 0 & I_{q}
\end{array}\right] .
\end{aligned}
$$

Remark 1: Note that the system (44) - (45) is not a physical system; rather it is a 'fictitious' system that treats the faults $\xi$ and disturbances $f$ as its unknown inputs. The key point is that it possesses a measurable 'output' which is $\bar{y}$; hence an observer can be constructed for (44)-(45) to estimate $f$. This approach of estimating faults using a measurable output of a fictitious system is not new and has been used in $[10,14]$.

Remark 2: The purpose of the filtering in (42)-(43) is to achieve the structure in (44) where the fault and disturbance vectors have been forced to be in the 'state equation' (44), which is the framework where the fault reconstruction technique can be applied to. This technique has been widely used in the published literature, for example [15,7]. If the filters have not been used and $w_{1}, w_{2}$ have been used directly as the output $\bar{y}$, then there will be faults and disturbances in the 'output equation' which is not the structure where the fault reconstruction technique can be applied to.

Further expanding $\bar{A}, \bar{M}, \bar{C}, \bar{Q}$ using (28)-(31) and (34) to get

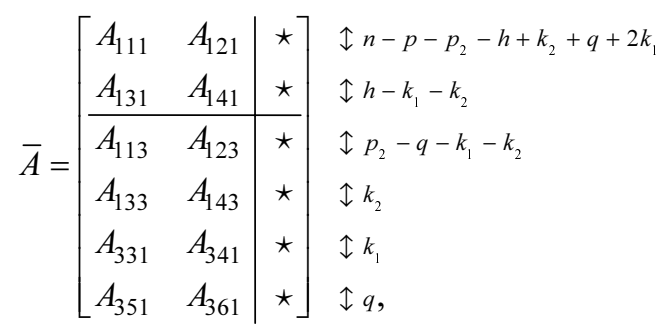




$$
\begin{aligned}
& \bar{Q}=\left[\begin{array}{cc}
0 & 0 \\
Q_{111} & 0 \\
\hline 0 & 0 \\
Q_{112} & 0 \\
0 & Q_{22} \\
0 & 0
\end{array}\right], \bar{M}=\left[\begin{array}{c}
0 \\
0 \\
M_{o}
\end{array}\right], \\
& \bar{C}=\left[\begin{array}{cc|cccc}
0 & 0 & A_{3112} & 0 & 0 & 0 \\
0 & 0 & A_{3122} & A_{322} & 0 & 0 \\
0 & 0 & 0 & 0 & I & 0 \\
0 & 0 & 0 & 0 & 0 & I
\end{array}\right] \uparrow p_{2}-q-k_{1}-k_{2} \\
& \uparrow q .
\end{aligned}
$$

Note that $Q_{111}, Q_{112}$ will form a square and invertible matrix. Therefore there exists a matrix $\bar{T}_{\xi} \in$ $\mathbb{R}^{\left(h-k_{1}\right) \times\left(h-k_{1}\right)}$ such that

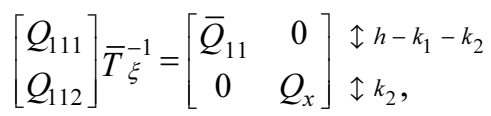

where $\bar{Q}_{11}, Q_{x}$ are square and invertible. By performing the a transformation on $\xi \mapsto\left[\begin{array}{cc}\bar{T} \xi & 0 \\ 0 & I_{k_{1}}\end{array}\right] \xi$ results in

$$
\begin{aligned}
& \bar{Q} \mapsto \bar{Q}\left[\begin{array}{cc}
\bar{T}_{\xi} & 0 \\
0 & I_{k_{1}}
\end{array}\right]^{-1}=\left[\begin{array}{l}
\bar{Q}_{1} \\
\bar{Q}_{2}
\end{array}\right]
\end{aligned}
$$

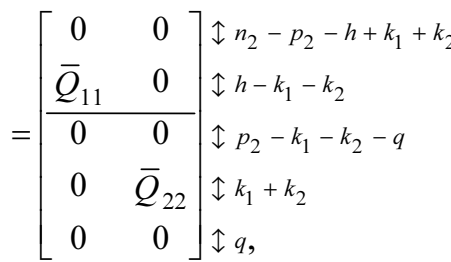

$$
\begin{aligned}
& \bar{Q}_{22}:=\left[\begin{array}{cc}
Q_{x} & 0 \\
0 & Q_{22}
\end{array}\right] .
\end{aligned}
$$

The matrices $\bar{A}, \bar{C}, \bar{M}$ remain unaltered by the transformation, but can also be re-expressed so that they are partitioned conformably with $\bar{Q}$ in (53), as follows

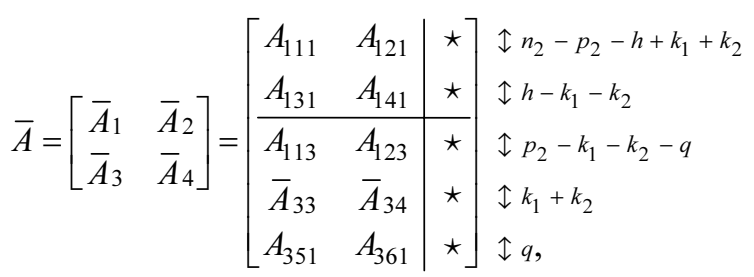

$$
\begin{aligned}
& \bar{M}=\left[\begin{array}{c}
0 \\
\bar{M}_{2}
\end{array}\right]=\left[\begin{array}{c}
0 \\
\hline 0 \\
\bar{M}_{o}
\end{array}\right] \begin{array}{l}
\mathfrak{l} n_{2}-p_{2} \\
\uparrow p_{2}-q, \\
\uparrow q
\end{array}
\end{aligned}
$$

where $\star$ are matrices of $p_{2}$ columns that do not play any role in the following analysis.

Notice that the structure of $\bar{A}, \bar{M}, \bar{C}, \bar{Q}$ in (53)-(55) is identical to the structure of $A, M, C, Q$ in (3)-(6).
Therefore, using the results of $[8,9]$, it is possible to achieve DDFR if the following conditions are satisfied C1. $\left(\bar{A},\left[\begin{array}{ll}\bar{M} & \bar{Q}\end{array}\right], \bar{C}\right)$ is minimum phase

C2. $\operatorname{rank}\left(A_{123}\right)=\operatorname{rank}\left[\begin{array}{l}A_{121} \\ A_{123} \\ A_{361}\end{array}\right]$

C3. The first observer has a stable sliding motion.

Then a secondary sliding mode observer [3] can be implemented on the system (44)-(45) similar to what was done in Section 2.1. Let $\bar{L}=\left[\begin{array}{lll}\bar{L}_{11} & \bar{L}_{12} & 0 \\ \bar{L}_{13} & \bar{L}_{14} & 0\end{array}\right]$ be such that $\quad \bar{A}_{1}+\bar{L}_{3} \quad$ is stable (where $\bar{L} \in \mathbb{R}^{\left(n_{2}-p_{2}\right) \times p_{2}}$, $\left.\bar{L}_{13} \in \mathbb{R}^{\left(h-k_{1}-k_{2}\right) \times\left(p_{2}-k_{1}-k_{2}-q\right)}, \quad \bar{L}_{14} \in \mathbb{R}^{\left(h-k_{1}-k_{2}\right) \times\left(k_{1}+k_{2}\right)}\right)$ and $\bar{v}_{e q}$ be the equivalent output error injection required to maintain sliding motion for the second observer. Then let there be a pair $\bar{P}_{o} \in \mathbb{R}^{p_{2} \times p_{2}}$, $\bar{G}_{l} \in \mathbb{R}^{n_{2} \times p_{2}}$ such that the condition in Lemma 1 is satisfied. Then define the fault reconstruction signal $\hat{f}:=\bar{W}_{312}^{-1} \bar{P}_{o}^{-1}$ where $\bar{W}:=\left[\begin{array}{lll}\bar{W}_{1} & 0 & M_{o}^{-1}\end{array}\right]$ and do the necessary re-arrangements as in Section 2.1 ; it results in the fault reconstruction error (from the second observer) being excited through a state-space system with the triple $(\bar{A}, \bar{B}, \bar{C})$ (in the same way as (18) - (23)), where

$$
\begin{aligned}
& \overline{\mathscr{A}}:=\bar{A}_{1}+\overline{L A}_{3} \equiv\left[\begin{array}{cc}
\overline{\mathscr{A}}_{1} & \overline{\mathscr{A}}_{2} \\
\bar{A}_{3} & \overline{\mathscr{A}}_{4}
\end{array}\right], \\
& \overline{\mathscr{B}}:=\bar{Q}_{1}+\bar{L}_{\bar{Q}_{2}}=\left[\begin{array}{cc}
0 & \bar{L}_{12} \bar{Q}_{22} \\
\bar{Q}_{11} & \bar{L}_{14} \bar{Q}_{22}
\end{array}\right] \equiv\left[\begin{array}{cc}
0 & \overline{\mathscr{B}}_{2} \\
\overline{\mathscr{B}}_{3} & \overline{\mathscr{B}}_{4}
\end{array}\right], \\
& \overline{\mathscr{C}} \equiv\left[\begin{array}{ll}
\overline{\mathscr{C}}_{1} & \overline{\mathscr{C}}_{2}
\end{array}\right],
\end{aligned}
$$

where $\overline{\mathscr{J}}_{1}=A_{111}+\bar{L}_{11} A_{113}+\bar{L}_{12} \bar{A}_{33}, \bar{\nearrow}_{2}=A_{121}+\bar{L}_{11} A_{123}^{*}$ $+\bar{L}_{12} \bar{A}_{34}, \quad \overline{\mathscr{A}}_{3}=A_{131}+\bar{L}_{13} A_{113}+\bar{L}_{14} \bar{A}_{33}, \quad \bar{A}_{4}=A_{141}+$ $\bar{L}_{13} A_{123}+\bar{L}_{14} \bar{A}_{34}, \quad \overline{\mathscr{C}}_{1}=\bar{W}_{1} A_{113}+M_{o}^{-1} A_{351} \quad$ and $\quad \overline{\mathscr{C}}_{2}=$ $\bar{W}_{1} A_{123}+M_{o}^{-1} A_{361}$.

If Conditions $\mathrm{C} 1-\mathrm{C} 3$ are satisfied, then $\bar{W}_{1}, \bar{L}_{11}$, $\bar{L}_{12}, \bar{L}_{13}, \bar{L}_{14}$ can be chosen such that the system $(\overline{\mathscr{C}}, \overline{\mathscr{B}}, \bar{C})$ will be made zero (see $[8,9])$.

\section{EXISTENCE CONDITIONS IN TERMS OF ORIGINAL SYSTEM MATRICES}

This section seeks to recast Conditions C1-C3 in terms of the original system matrices so that it is easy for the user to immediately determine from the outset whether or not it is possible to achieve DDFR using the scheme in this paper.

\subsection{Condition $\mathrm{C} 1$}

Proposition 2: The systems $(\bar{A},[\bar{M} \quad \bar{Q}], \bar{C})$ and $\left(A,\left[\begin{array}{ll}M & Q\end{array}\right], C\right)$ have the same invariant zeros. 
Proof: The Rosenbrock system matrix [12] of $(A,[M$ $Q], C)$ is as follows

$$
U_{1}(s):=\left[\begin{array}{ccc}
s I-A & -M & -Q \\
C & 0 & 0
\end{array}\right]
$$

and the invariant zeros of a system are the values of $s$ that make its Rosenbrock matrix lose rank [12]. Substituting for $A, M, Q, C$ from (28)-(31) results in

$$
U_{1}(s)=\left[\begin{array}{c|c}
U_{1 a} & U_{1 b} \\
\hline U_{1 c} & U_{1 d}
\end{array}\right],
$$

where

$$
\begin{aligned}
& U_{1 a}=\left[\begin{array}{ccccc}
s I-A_{111} & -A_{121} & -A_{112} & -A_{122} & \star \\
-A_{131} & s I-A_{141} & -A_{132} & -A_{142} & \star \\
-A_{113} & -A_{123} & s I-A_{114} & -A_{124} & \star \\
-A_{133} & -A_{143} & -A_{134} & s I-A_{144} & \star \\
0 & 0 & 0 & 0 & \star \\
0 & 0 & -A_{3112} & 0 & \star \\
0 & 0 & -A_{3122} & -A_{322} & \star \\
-A_{331} & -A_{341} & -A_{332} & -A_{342} & \star \\
-A_{351} & -A_{361} & -A_{352} & -A_{362} & \star
\end{array}\right], \\
& U_{1 b}=\left[\begin{array}{ccc}
0 & 0 & 0 \\
0 & -Q_{111} & 0 \\
0 & 0 & 0 \\
0 & -Q_{112} & 0 \\
0 & 0 & 0 \\
0 & 0 & 0 \\
0 & 0 & 0 \\
0 & 0 & -Q_{22} \\
-M_{o} & 0 & 0
\end{array}\right] \\
& U_{1 c}=\left[\begin{array}{lllll}
0 & 0 & 0 & 0 & \breve{C}_{2}
\end{array}\right], \quad U_{1 d}=\left[\begin{array}{lll}
0 & 0 & 0
\end{array}\right] \text {. } \\
& \text { Since } Q_{22}, M_{o}, \breve{C}_{2},\left[\begin{array}{l}
Q_{111} \\
Q_{112}
\end{array}\right] \text { and }\left[\begin{array}{cc}
A_{3112} & 0 \\
A_{3122} & A_{322}
\end{array}\right] \text { are }
\end{aligned}
$$
square and invertible, then $U_{1}(s)$ loses rank if and only if $U_{12}(s)$ loses rank, where

$$
U_{12}(s):=\left[\begin{array}{cc}
s I-A_{111} & -A_{121} \\
-A_{113} & -A_{123}
\end{array}\right] .
$$

Using (51)-(55), the Rosenbrock matrix of $(\bar{A},[\bar{M}$ $\bar{Q}], \bar{C})$ is as follows

$$
\begin{aligned}
& U_{1}(s):= \\
& {\left[\begin{array}{ccc|ccc}
s I-A_{111} & -A_{121} & \star & 0 & 0 & 0 \\
-A_{131} & s I-A_{141} & \star & 0 & -\bar{Q}_{11} & 0 \\
-A_{113} & -A_{123} & \star & 0 & 0 & 0 \\
-\bar{A}_{33} & -\bar{A}_{34} & \star & 0 & 0 & -\bar{Q}_{22} \\
-A_{351} & -A_{361} & \star & -M_{o} & 0 & 0 \\
\hline 0 & 0 & \breve{A}_{322} & 0 & 0 & 0
\end{array}\right] .}
\end{aligned}
$$

Since $\bar{Q}_{11}, \bar{Q}_{22}, M_{o}$ and $\breve{A}_{322}$ are square and invertible, then $U_{2}(s)$ loses rank if and only if $U_{22}(s)$ loses rank, where

$$
U_{22}(s):=\left[\begin{array}{cc}
s I-A_{111} & -A_{121} \\
-A_{113} & -A_{123}
\end{array}\right] .
$$

Therefore, it is straightforward to show that $U_{21}(s)$ $=U_{22}(s)$ and the proof is complete.

Therefore, $\mathrm{C} 1$ can be recasted in terms of the original system matrices as $\left(A,\left[\begin{array}{ll}M & Q\end{array}\right], C\right)$ being minimum phase, which is identical to Condition $\mathrm{B} 1$.

\subsection{Condition $\mathrm{C} 2$}

Proposition 3: Define $A_{o}:=A-K C$, where $K:=$ $K_{1} C_{2}^{-1}$ with $K_{1}$ being the last $p$ columns of $A$ (therefore $A_{o}$ is identical to $A$ except that the last $p$ columns of $A_{o}$ are all zero). Then it can be shown that

$$
\operatorname{rank}\left(X_{1}\right)=\operatorname{rank}\left(X_{10}\right), \operatorname{rank}\left(X_{2}\right)=\operatorname{rank}\left(X_{20}\right),
$$

where

$$
\begin{aligned}
X_{10}:= & {\left[\begin{array}{cccccc}
A Q & 0 & 0 & Q & 0 & 0 \\
C Q & 0 & 0 & 0 & 0 & 0 \\
C A_{o} Q & C Q & 0 & 0 & C M & 0 \\
C A_{o}^{2} Q & C A_{o} Q & C Q & 0 & C A_{o} M & C M
\end{array}\right], } \\
X_{20}:= & {\left[\begin{array}{ccccc}
C Q & 0 & 0 & 0 & 0 \\
C A_{o} Q & C Q & 0 & C M & 0 \\
C A_{o}^{2} Q & C A_{o} Q & C Q & C A_{o} M & C M
\end{array}\right] }
\end{aligned}
$$

Proof: Define the following square and invertible matrices

$$
\begin{aligned}
T_{10}: & =\left[\begin{array}{cccc}
I_{n} & 0 & 0 & 0 \\
0 & I_{p} & 0 & 0 \\
0 & -C K & I_{p} & 0 \\
0 & -C A K & -C K & I_{p}
\end{array}\right], \\
T_{20}: & =\left[\begin{array}{ccc}
I_{p} & 0 & 0 \\
-C K & I_{p} & 0 \\
-C A K & -C K & I_{p}
\end{array}\right] .
\end{aligned}
$$

It is straightforward to show that $X_{1}=T_{10} X_{10}$ and $X_{2}=T_{20} X_{20}$. Since $T_{10}$ and $T_{20}$ are both square and invertible, then $\operatorname{rank}\left(X_{1}\right)=\operatorname{rank}\left(X_{10}\right), \operatorname{rank}\left(X_{2}\right)=$ $\operatorname{rank}\left(X_{20}\right)$

Proposition 4: It can be shown that

$$
\begin{aligned}
\operatorname{rank}\left(X_{1}\right)-\operatorname{rank}\left(X_{2}\right)= & \operatorname{rank}\left[\begin{array}{l}
A_{121} \\
A_{123} \\
A_{361}
\end{array}\right] \\
& -\operatorname{rank}\left(A_{123}\right)+\operatorname{rank}(Q) .
\end{aligned}
$$


Proof: Using (28)-(33), the following can be established:

$$
\begin{aligned}
& C M=\breve{C}_{2}\left[\begin{array}{c}
0 \\
M_{o}
\end{array}\right] \mathfrak{\downarrow} p, \quad
\end{aligned}
$$

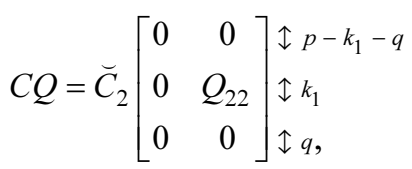

$$
\begin{aligned}
& C A_{o} Q=\check{C}_{2}\left[\begin{array}{ccc}
0 & 0 & 0 \\
0 & A_{322} & 0 \\
A_{341} & A_{342} & 0 \\
A_{361} & A_{362} & 0
\end{array}\right] X_{3} \text {, }
\end{aligned}
$$

where $X_{3}=\left[\begin{array}{cc}R_{3}^{-1} Q_{11} & 0 \\ 0 & I_{k_{1}}\end{array}\right]$.

$$
C A_{o}^{2} Q=\breve{C}_{2}\left[\begin{array}{ccc}
I_{p-p_{2}} & 0 & 0 \\
0 & \breve{A}_{312} & 0 \\
0 & 0 & I_{q+k_{1}}
\end{array}\right]\left[\begin{array}{cc|c}
0 & 0 & 0 \\
\hline A_{123} & A_{124} & 0 \\
A_{143} & A_{144} & 0 \\
\hline \star & \star & 0
\end{array}\right] X_{3} \text {, }
$$

where $\star$ are matrices with $q+k_{1}$ rows that do not play any role in the following analysis.

Then, define

$$
\begin{aligned}
& C_{10}:=\operatorname{diag}\left\{I_{n}, \breve{C}_{2}, \breve{C}_{2}, \breve{R}\right\}, \\
& Q_{10}:=\operatorname{diag}\left\{R_{3}^{-1} Q_{11}, I_{k_{1}}, R_{3}^{-1} Q_{11}, I_{k_{1}}, I_{2 h+2 q}\right\},
\end{aligned}
$$

where $\breve{R}:=\breve{C}_{2} \operatorname{diag}\left\{I_{p-p_{2}}, \breve{A}_{312}, I_{q+k_{1}}\right\}$. It can be shown that $X_{10}$ from Proposition 3 can be expanded to be

$$
C_{10}\left[\begin{array}{c|c|c}
\mathscr{X}_{a} & \mathscr{X}_{b} & 0 \\
\hline \mathscr{X}_{c} & 0 & \mathscr{X}_{d}
\end{array}\right] Q_{10},
$$

where

$$
\begin{aligned}
& \mathscr{X}_{a}=\left[\begin{array}{ccc|ccc|cc}
A_{121} & A_{122} & \star & 0 & 0 & 0 & 0 & 0 \\
A_{141} & A_{142} & \star & 0 & 0 & 0 & 0 & 0 \\
A_{123} & A_{124} & \star & 0 & 0 & 0 & 0 & 0 \\
A_{143} & A_{144} & \star & 0 & 0 & 0 & 0 & 0 \\
0 & 0 & \star & 0 & 0 & 0 & 0 & 0 \\
0 & A_{322} & \star & 0 & 0 & 0 & 0 & 0 \\
A_{341} & A_{342} & \star & 0 & 0 & 0 & 0 & 0 \\
A_{361} & A_{362} & \star & 0 & 0 & 0 & 0 & 0
\end{array}\right], \\
& \mathscr{P}_{b}=\left[\begin{array}{cc}
0 & 0 \\
Q_{111} & 0 \\
0 & 0 \\
Q_{112} & 0 \\
0 & 0 \\
0 & 0 \\
0 & Q_{22} \\
0 & 0
\end{array}\right],
\end{aligned}
$$

$$
\begin{aligned}
\mathscr{R}_{c} & =\left[\begin{array}{ccc|ccc|cc}
0 & 0 & 0 & 0 & 0 & 0 & 0 & 0 \\
0 & 0 & Q_{22} & 0 & 0 & 0 & 0 & 0 \\
0 & 0 & 0 & 0 & 0 & 0 & 0 & 0 \\
\hline 0 & 0 & 0 & 0 & 0 & 0 & 0 & 0 \\
0 & A_{322} & 0 & 0 & 0 & 0 & 0 & 0 \\
A_{341} & A_{342} & 0 & 0 & 0 & Q_{22} & 0 & 0 \\
A_{361} & A_{362} & 0 & 0 & 0 & 0 & 0 & 0 \\
\hline 0 & 0 & 0 & 0 & 0 & 0 & 0 & 0 \\
A_{123} & A_{124} & 0 & 0 & 0 & 0 & 0 & 0 \\
A_{143} & A_{144} & 0 & 0 & A_{322} & 0 & 0 & 0 \\
\star & \star & 0 & A_{341} & A_{342} & 0 & 0 & Q_{22} \\
\star & \star & 0 & A_{361} & A_{362} & 0 & 0 & 0
\end{array}\right], \\
\mathscr{X}_{d} & =\left[\begin{array}{c|c}
0 & 0 \\
0 & 0 \\
0 & 0 \\
\hline 0 & 0 \\
0 & 0 \\
M_{o} & 0 \\
\hline 0 & 0 \\
0 & 0 \\
0 & 0 \\
0 & 0 \\
0 & M_{o}
\end{array}\right] .
\end{aligned}
$$

Since $C_{10}, Q_{10}$ are square and invertible, and recalling that $\operatorname{rank}\left(Q_{22}\right)=k_{1}, \operatorname{rank}\left(M_{o}\right)=q, \operatorname{rank}\left(A_{322}\right)=k_{2}$ and $\operatorname{rank}\left[\begin{array}{l}Q_{111} \\ Q_{112}\end{array}\right]=h-k_{1}$, then it can be shown that

$$
\begin{aligned}
\operatorname{rank}\left(X_{10}\right) & =2 q+3 k_{1}+2 k_{2}+h+\operatorname{rank}\left[\begin{array}{l}
A_{121} \\
A_{123} \\
A_{361} \\
A_{123}
\end{array}\right] \\
& =2 q+3 k_{1}+2 k_{2}+h+\operatorname{rank}\left[\begin{array}{l}
A_{121} \\
A_{123} \\
A_{361}
\end{array}\right] .
\end{aligned}
$$

Then, define

$$
\begin{aligned}
C_{20} & :=\operatorname{diag}\left\{\breve{C}_{2}, \breve{C}_{2}, \breve{R}\right\}, \\
Q_{20} & :=\operatorname{diag}\left\{R_{3}^{-1} Q_{11}, I_{k_{1}}, R_{3}^{-1} Q_{11}, I_{k_{1}}, I_{h+2 q}\right\} .
\end{aligned}
$$

It can be shown that $X_{20}$ from Proposition 224 can be expanded to be

$$
C_{20}\left[\mathscr{C l}_{c} \mathscr{X}_{d}\right] Q_{20} \text {. }
$$

Since $C_{20}, Q_{20}$ are square and invertible, then it follows that 


$$
\operatorname{rank}\left(X_{20}\right)=2 q+3 k_{1}+2 k_{2}+\operatorname{rank}\left(A_{123}\right) .
$$

Then from (64) and (65), using the result of Proposition 3, and recalling that $\operatorname{rank}(Q)=h$, the proof is complete.

Hence, from (60), $\operatorname{rank}\left[\begin{array}{l}A_{121} \\ A_{123} \\ A_{361}\end{array}\right]=\operatorname{rank}\left(A_{123}\right) \Leftrightarrow \operatorname{rank}$ $\left(X_{1}\right)-\operatorname{rank}\left(X_{2}\right)=\operatorname{rank}(Q)$ which is identical to Condition B2.

\subsection{Condition $\mathrm{C} 3$}

Proposition 5: A stable sliding motion exists for the first observer if $\left(A,\left[\begin{array}{ll}M & Q\end{array}\right], C\right)$ is minimum phase.

Proof: From the structure of $L$ in (34), it is clear that $\left(\breve{A}_{1}+L \breve{A}_{3}\right)$ is stable if and only if the pair $\left(\breve{A}_{1}, \breve{A}_{31}\right)$ is detectable.

From the Popov-Hautus-Rosenbrock (PHR) rank test [12], the unobservable modes of $\left(\breve{A}_{1}, \breve{A}_{31}\right)$ are the values of $s$ that make the following matrix pencil lose rank

$$
U_{3}(s)=\left[\begin{array}{c}
s I-\breve{A}_{1} \\
\breve{A}_{31}
\end{array}\right] .
$$

Substituting for the pair $\left(\breve{A}_{1}, \breve{A}_{31}\right)$ from (28) results in

$$
U_{3}(s)=\left[\begin{array}{cccc}
s I-A_{111} & -A_{121} & -A_{112} & -A_{122} \\
-A_{131} & s I-A_{141} & -A_{132} & -A_{142} \\
-A_{113} & -A_{123} & s I-A_{114} & -A_{124} \\
-A_{133} & -A_{143} & -A_{134} & s I-A_{144} \\
\hline 0 & 0 & 0 & 0 \\
0 & 0 & -A_{3112} & 0 \\
0 & 0 & -A_{3122} & -A_{322}
\end{array}\right] .
$$

It is then clear that $U_{3}(s)$ loses rank if and only if $U_{32}(s)$ loses rank, where

$$
U_{32}(s)=\left[\begin{array}{cc}
s I-A_{111} & -A_{121} \\
-A_{131} & s I-A_{141} \\
-A_{113} & -A_{123} \\
-A_{133} & -A_{143}
\end{array}\right] .
$$

It is easy to see that if $U_{12}(s)$ is full rank, then $U_{32}(s)$ is also full rank. If $\left(\breve{A}_{1}, \breve{A}_{31}\right)$ is minimum phase, then $U_{12}(s)$ is full rank (and $U_{32}(s)$ will also be full rank) when $s \in \mathbb{C}_{+}$which implies that any values of $s$ that make $U_{32}(s)$ lose rank will be stable, resulting in the detectability of $\left(\breve{A}_{1}, \breve{A}_{31}\right)$, and as a consequence a stable sliding motion existing for the first observer.

Therefore, the results in this section show that Conditions C1-C3 (which guarantee DDFR using the 2observer structure in this paper) are guaranteed by Conditions B1 - B2. Hence Theorem 1 is proven.

Remark 3: Notice that Condition $B 2$ is less restrictive than Condition A2 because Condition A2 implies that $A_{121}=0, \quad A_{123}=0, \quad A_{361}=0$ whereas Condition B2 implies that $\operatorname{rank}\left(A_{123}\right)=\operatorname{rank}\left[\begin{array}{lll}A_{121}^{T} & A_{123}^{T} & A_{361}^{T}\end{array}\right]^{T}$ which is obviously a weaker condition. Recall that for the work that uses only one observer [8,9], DDFR can be guaranteed if A2 is satisfied. Therefore, the 2-observer algorithm in this paper is able to achieve DDFR for a wider class of systems compared to using only one observer as in [8,9].

Remark 4: The ability of the second observer to achieve DDFR does not depend on the design of the first observer, namely $G_{l}$ and $L_{1}$. This is seen from the fact that the conditions B1 and B2 are in terms of the original system matrices. Therefore, it is possible for the designer to know from the outset whether DDFR can be achieved using the 2-observer method in this paper, without having to a-priori design the first observer.

\section{SIMULATION EXAMPLE}

The method described in this paper will be demonstrated using a simulation example. Consider a $3 \mathrm{rd}$ order general nonlinear system described as:

$$
\frac{d^{3}}{d t^{3}} \theta+a_{1} \frac{d^{2}}{d t^{2}} \theta+a_{2} \frac{d}{d t} \theta+a_{3} \theta+\xi=u,
$$

where $\theta$ is the position and $u$ is the measurable control input. For simplicity, let $u \equiv 0$. Without loss of generality, the term $\xi$ will encapsulate any disturbances or nonlinearities present in the system. For example, for a nonlinear uncertain system represented by $\frac{d^{3}}{d t^{3}} \theta+a_{1} \frac{d^{2}}{d t^{2}} \theta+a_{2} \frac{d}{d t} \theta+a_{3} \theta+\sin (\theta)+\theta^{2}+d=u$ where $d$ is an external disturbance, then $\xi=\sin (\theta)+\theta^{2}+d$.

Let $a_{1}=2, a_{2}=3, a_{3}=4$. Assume that $\theta, \dot{\theta}, \ddot{\theta}$ are measurable. However, let the sensors of $\dot{\theta}, \ddot{\theta}$ be assumed to be faulty. Hence the sensor equations can be written as

$$
y_{1}=\ddot{\theta}+f_{\text {heta }}, y_{2}=\dot{\theta}+f_{\dot{\theta}}, y_{3}=\theta .
$$

Filter the signals $y_{1}, y_{2}$ to respectively generate $y_{f, 1}$, $y_{f, 2}$ as follows:

$$
\begin{aligned}
& \dot{y}_{f, 1}=-y_{f, 1}+y_{1}=-y_{f, 1}+\ddot{\theta}+f_{\ddot{\theta}}, \\
& \dot{y}_{f, 2}=-y_{f, 2}+y_{2}=-y_{f, 2}+\dot{\theta}+f_{\dot{\theta}} .
\end{aligned}
$$

Combine (67), (68) and (69) to obtain the following state-space system in the framework of (1)-(2) where 


$$
\begin{aligned}
& \breve{A}=\left[\begin{array}{cc|ccc}
0 & 1 & 0 & 0 & 0 \\
-a_{2} & -a_{1} & -a_{3} & 0 & 0 \\
\hline 1 & 0 & 0 & 0 & 0 \\
0 & 1 & 0 & -1 & 0 \\
1 & 0 & 0 & 0 & -1
\end{array}\right], \quad \breve{M}=\left[\begin{array}{ll}
0 & 0 \\
0 & 0 \\
\hline 0 & 0 \\
1 & 0 \\
0 & 1
\end{array}\right], \\
& \breve{Q}=\left[\begin{array}{l}
0 \\
1 \\
\hline 0 \\
0 \\
0
\end{array}\right], \quad \breve{C}=\left[\begin{array}{ll|ccc}
0 & 0 & 1 & 0 & 0 \\
0 & 0 & 0 & 1 & 0 \\
0 & 0 & 0 & 0 & 1
\end{array}\right] .
\end{aligned}
$$

Notice that (69)-(70) is already in structure of (3)-(6). From the parameters given above, it can be established that $n=5, p=3, q=2, h=1, C Q=0 \Rightarrow k_{1}=0$. Comparing with (7), it is clear that $A_{32}^{*}=0, A_{36}^{*}=\left[\begin{array}{ll}1 & 0\end{array}\right]^{T}$ hence Condition A2 is not satisfied and it is not possible to guarantee DDFR using one observer as described in Section 2.1. Besides, by further analyzing the work in $[9,8]$, it is found that when $A_{32}^{*}=0, A_{36}^{*} \neq 0 \infty$ it is impossible to achieve DDFR using one observer. However, Conditions B1 and B2 are satisfied, hence it is possible to achieve DDFR using the 2-observer method in this paper. The following choice of coordinate transform $Z$

$$
Z=\left[\begin{array}{lll}
0 & 1 & 0 \\
1 & 0 & 0 \\
0 & 0 & I_{3}
\end{array}\right]
$$

will cause $A$ to have the structure in (28) with $A_{322}=$ $\phi$ (empty matrix) and $A_{3112}=1$, which is full rank.

\subsection{Design of observer 1}

It is desired that $\lambda\left(\breve{A}_{1}+L \breve{A}_{3}\right)=-2,-3$, hence the appropriate choice of $L_{1}$ is $L_{1}=\left[\begin{array}{c}3 \\ -3\end{array}\right]$. The observer gains $G_{l}, P_{o}$ are designed using the method in [3] where $\lambda\left(\breve{A}_{1}+L \breve{A}_{3}\right)$ are a subset of $\lambda\left(A-G_{l} C\right)$. From [3], by choosing the remaining eigenvalues of $A-G_{l} C$ to be $-3,-4,-5$, the gain $G_{l}$ can be obtained, and a suitable choice of $P_{o}$ was found to be $P_{o}=I_{3}$. From the values of $P_{o}$ and $L$ obtained, the gain $G_{n}$ can be determined from (12). The following are the calculated values of the gains (that will guarantee sliding motion of the first observer):

$$
G_{l}=\left[\begin{array}{ccc}
-16 & 0 & 0 \\
6 & 0 & 0 \\
6 & 0 & 0 \\
-3 & 3 & 0 \\
3 & 0 & 4
\end{array}\right], G_{n}=\left[\begin{array}{ccc}
-3 & 0 & 0 \\
3 & 0 & 0 \\
1 & 0 & 0 \\
0 & 1 & 0 \\
0 & 0 & 1
\end{array}\right] .
$$

\subsection{Design of observer 2}

Choosing $\alpha_{1}=\alpha_{2}=1$ results in the following matrices

$$
\begin{aligned}
& \bar{A}=\left[\begin{array}{c|ccc}
-2 & 0 & 0 & 0 \\
\hline 1 & -3 & 0 & 0 \\
1 & 0 & -1 & 0 \\
0 & 1 & 0 & -1
\end{array}\right], \bar{M}=\left[\begin{array}{ll}
0 & 0 \\
0 & 0 \\
1 & 0 \\
0 & 1
\end{array}\right], \\
& \bar{C}=\left[\begin{array}{l|lll}
0 & 1 & 0 & 0 \\
0 & 0 & 1 & 0 \\
0 & 0 & 0 & 1
\end{array}\right], \bar{Q}=\left[\begin{array}{l}
0 \\
1 \\
0 \\
0
\end{array}\right] .
\end{aligned}
$$

Comparing $\bar{A}, \bar{M}, \bar{C}, \bar{Q}$ with (53)-(55), it can be seen that $\bar{Q}_{11}=1, \bar{Q}_{22}=\phi$ empty matrix, $\bar{C}_{2}=I_{3}$ and $\bar{M}_{o}=I_{2}$, hence resulting in $A_{123}=1$ which has full column rank. As a consequence, Condition $\mathrm{C} 2$ is satisfied, which verifies the earlier fact that 2 observers are sufficient to achieve DDFR.

In designing the second observer, $\bar{L}_{1}=\bar{L}=\left[\begin{array}{lll}-1 & 0 & 0\end{array}\right]$ is chosen so that the reduced order sliding motion for the second observer has a pole at -3 . (See [8] on how the matrix $\bar{L}_{1}$ is designed to achieve DDFR for the second system (71)-(72)). The gains $\bar{G}_{l}$ and $\bar{P}_{o}$ are designed using the algorithm in [3]; the remaining eigenvalues of $\bar{A}-\bar{G}_{l} \bar{C}$ are specified to be $-4,-5,-6$, and $\bar{P}_{o}=I_{3}$ is an appropriate choice. The following gains are the resulting appropriate gains to guarantee a sliding motion:

$$
\bar{G}_{n}=\left[\begin{array}{lll}
1 & 0 & 0 \\
1 & 0 & 0 \\
0 & 1 & 0 \\
0 & 0 & 1
\end{array}\right], \bar{G}_{l}=\left[\begin{array}{lll}
2 & 0 & 0 \\
2 & 0 & 0 \\
1 & 4 & 0 \\
1 & 0 & 5
\end{array}\right] .
$$

Then choosing $\bar{W}_{1}=\left[\begin{array}{ll}-1 & 0\end{array}\right]^{T}$ to get $\bar{W}=\left[\begin{array}{ccc}-1 & 1 & 0 \\ 0 & 0 & 1\end{array}\right]$ results in DDFR being achieved.

\subsection{Simulation results}

Faults were injected into the faulty sensors, together with the disturbance $\xi$. The left subfigure of Figs. 2 and 3 show the faults, and Fig. 4 shows the disturbance $\xi$. The middle subfigure of Figs. 2 and 3 shows the reconstructions of the fault which are visually identical to the fault despite the presence of the disturbance $\xi$, confirming the achievement of DDFR. The right subfigures of Figs. 2 and 3 shows the fault reconstruction error $\hat{f}-f$, though non-zero ${ }^{1}$, is very small.

${ }^{1}$ This is due to the sigmoidal approximation to obtain $v_{e q}$ in (17) which will result in a small phase lag between the fault and the fault reconstruction. The bigger the value of $\delta$ in (17), the bigger will the phase lag be. 

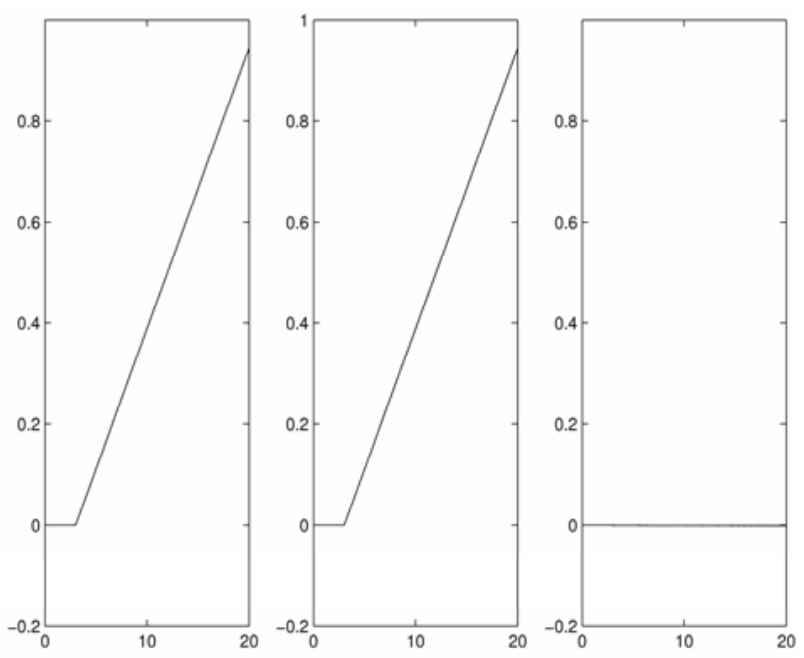

Fig. 2. The left subfigure is the fault in sensor 1 , the middle subfigure is its reconstruction, and the right subfigure is the fault reconstruction error.
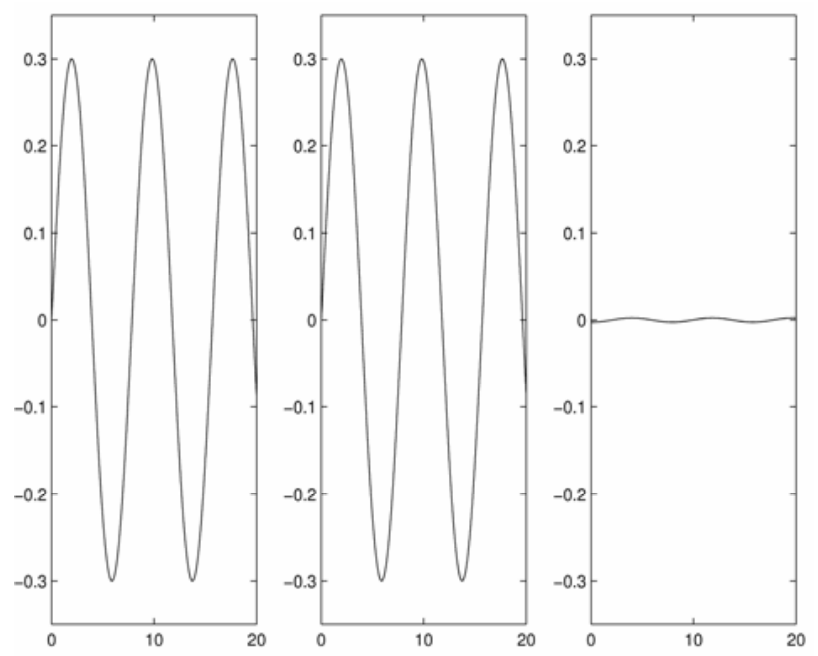

Fig. 3. The left subfigure is the fault in sensor 2, the middle subfigure is its reconstruction, and the right subfigure is the fault reconstruction error.

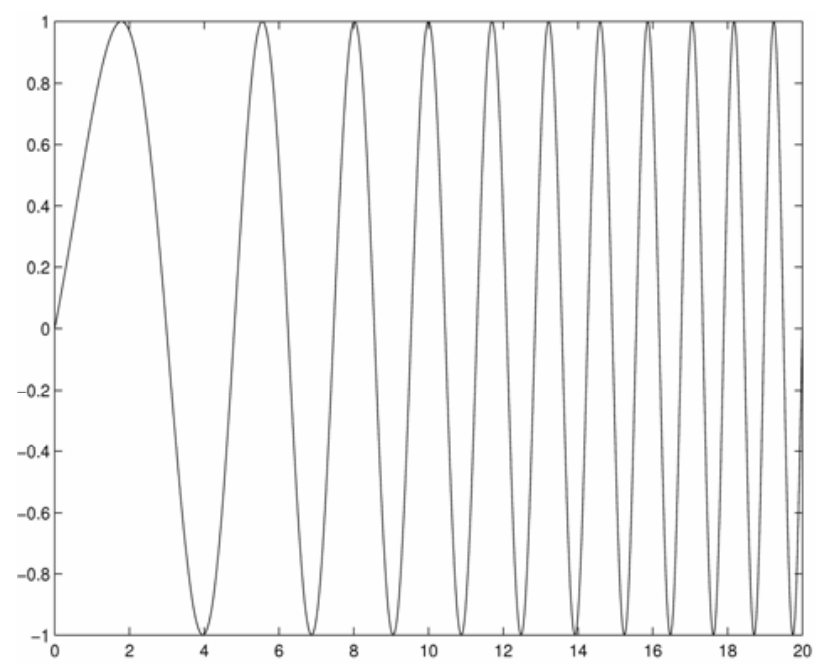

Fig. 4. The disturbance $\xi$.

\section{CONCLUSION}

This paper has presented new results in DDFR using two sliding mode observers in cascade. Measurable signals from the first observer are treated as the output for a second observer which will reconstruct the fault. It was found that by using two observers, DDFR could be achieved for a wider class of systems compared when just only one observer being used. This paper also investigated the conditions that guarantee the success of the scheme, which are found to be easily testable in terms of the original system matrices. This is very useful because the user can know from the outset whether the scheme in this paper is applicable to a particular system or not. A simulation example validates the claims made in this paper. The usage of a higher and general number of observers for further enhanced DDFR is under the authors' investigation.

\section{APPENDIX A}

\section{A.1. Proof of Proposition 1}

From [3], since N1 holds, then there exists a change of coordinates such that $(M, C, Q)$ can be written as

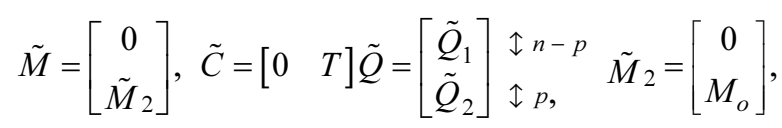

where $T$ is orthogonal and $M_{o}$ is square and invertible.

Let $\tilde{Q}_{2}=\left[\begin{array}{l}\tilde{Q}_{21} \\ \tilde{Q}_{22}\end{array}\right] \begin{aligned} & 1 p-q \\ & \uparrow q\end{aligned}$. Since $\operatorname{rank}(\tilde{C} \tilde{Q})=k_{1}$, then $\operatorname{rank}\left(\tilde{Q}_{2}\right)=k_{1}$ as $T$ is orthogonal. From the structure of $\tilde{C}$ in (A.1), it results in

$$
\tilde{C}\left[\begin{array}{cc}
\tilde{M} & \tilde{Q}
\end{array}\right]=T\left[\begin{array}{cc}
0 & \tilde{Q}_{21} \\
M_{o} & \tilde{Q}_{22}
\end{array}\right] .
$$

Assumption N2 then results in $\operatorname{rank}\left(\tilde{Q}_{21}\right)=k_{1}$ and hence

$$
\operatorname{rank}\left(\tilde{Q}_{21}\right)=\operatorname{rank}\left[\begin{array}{l}
\tilde{Q}_{21} \\
\tilde{Q}_{22}
\end{array}\right] .
$$

Therefore, there exists a matrix $\tilde{Q}_{21}^{\dagger}$ such that $\tilde{Q}_{21}^{\dagger} Q_{21}=R^{T}\left[\begin{array}{cc}0 & 0 \\ 0 & I_{k_{1}}\end{array}\right] R$ where $R \quad$ is an orthogonal matrix. It then follows that

$$
-\tilde{Q}_{22} \tilde{Q}_{21}^{\dagger} \tilde{Q}_{21}+\tilde{Q}_{22}=0
$$

Hence, applying the following change of coordinates $x \mapsto T_{\text {pre }} x$ where

$$
T_{\text {pre }}:=\left[\begin{array}{c|cc}
I & 0 & 0 \\
\hline 0 & I & 0 \\
0 & -\tilde{Q}_{22} \tilde{Q}_{21}^{\dagger} & I
\end{array}\right] \begin{aligned}
& \\
& \downarrow n-p \\
&
\end{aligned}
$$


and the structures in (3)-(4) are achieved.

Since $\operatorname{rank}\left(\tilde{Q}_{21}\right)=k_{1}$, there exists orthogonal matrices $N_{1} \in \mathbb{R}^{(n-p) \times(n-p)}, N_{2} \in \mathbb{R}^{(p-q) \times(p-q)}$ such that

$$
\left[\begin{array}{cc}
N_{1} & 0 \\
0 & N_{2}
\end{array}\right]\left[\begin{array}{c}
\tilde{Q}_{1} \\
\tilde{Q}_{21}
\end{array}\right] T_{\xi}^{-1}=\left[\begin{array}{cc}
0 & Q_{12} \\
Q_{11} & Q_{13} \\
\hline 0 & 0 \\
0 & Q_{22}
\end{array}\right] \begin{aligned}
& \\
& \uparrow n-p-h+k_{1} \\
& \uparrow p-k_{1}-q \\
& \uparrow k_{1},
\end{aligned}
$$

where $Q_{11}, Q_{22}$ are square and invertible.

Then define

$$
T_{1}=T_{1 a} T_{1 b} T_{1 c},
$$

where

$$
T_{1 a}=\left[\begin{array}{cc|ccc}
I_{n-p-h+k_{1}} & 0 & 0 & -Q_{12} Q_{22}^{-1} & 0 \\
0 & I_{h-k_{1}} & 0 & -Q_{13} Q_{22}^{-1} & 0 \\
\hline 0 & 0 & I_{p-k_{1}-q} & 0 & 0 \\
0 & 0 & 0 & I_{k_{1}} & 0 \\
0 & 0 & 0 & 0 & I_{q}
\end{array}\right],
$$$$
T_{1 b}=\left[\begin{array}{c|cc}
N_{1} & 0 & 0 \\
\hline 0 & N_{2} & 0 \\
0 & 0 & I_{q}
\end{array}\right],
$$$$
T_{1 c}=\left[\begin{array}{c|cc}
I_{n-p} & 0 & 0 \\
\hline 0 & I_{p-q} & 0 \\
0 & -\tilde{Q}_{22} \tilde{Q}_{21}^{\dagger} & 0
\end{array}\right] \text {, }
$$

and performing the transformations $x \mapsto T_{1} x, \quad \xi \mapsto T_{\xi} \xi$ results in $Q \mapsto T_{1} Q T_{\xi}^{-1}, M \mapsto T_{1} M, C \mapsto C T_{1}^{-1}$ and the structures in (3)-(6) are achieved.

\section{REFERENCES}

[1] S. P. Boyd, L. El-Ghaoui, E. Feron, and V. Balakrishnan, Linear Matrix Inequalities in Systems and Control Theory, SIAM, Philadelphia, 1994.

[2] J. Chen and R. J. Patton, Robust Model-Based Fault Diagnosis for Dynamic Systems, Kluwer Academic Publishers, 1999.

[3] C. Edwards and S. K. Spurgeon, "On the development of discontinuous observers," International Journal of Control, vol. 59, pp. 1211-1229, 1994.

[4] C. Edwards and S. K. Spurgeon, "A sliding mode observer based FDI scheme for the ship benchmark," European Journal of Control, vol. 6, pp. 341-356, 2000.

[5] C. Edwards, S. K. Spurgeon, and R. J. Patton, "Sliding mode observers for fault detection and isolation," Automatica, vol. 36, pp. 541-553, 2000.

[6] C. Edwards and C. P. Tan, "A comparison of sliding mode and unknown input observers for fault reconstruction," European Journal of Control, vol. 16, pp. 245-260, 2006.
[7] C. Edwards and C. P. Tan, "Fault tolerant control using sliding mode observers," Control Engineering Practice, vol. 14, pp. 897-908, 2006.

[8] K. Y. Ng, C. P. Tan, R. Akmeliawati, and C. Edwards, "Disturbance decoupled fault reconstruction using sliding mode observers," Proc. of the IFAC World Congress, Seoul, Korea, pp. 7215-7220, 2008.

[9] K. Y. Ng, C. P. Tan, R. Akmeliawati, and C. Edwards, "Disturbance decoupled fault reconstruction using sliding mode observers," Proc. of the 17th IFAC World Congress, Seoul, Korea, pp. 7215 7220, 2008.

[10] K. Y. Ng, C. P. Tan, C. Edwards, and Y. C. Kuang, "New results in robust actuator fault reconstruction in linear uncertain systems" International Journal of Robust and Nonlinear Control, vol. 17, pp. 12941319, 2007.

[11] R. J. Patton and J. Chen, "Optimal unknown input distribution matrix selection in robust fault diagnosis," Automatica, vol. 29, pp. 837-841, 1993.

[12] H. H. Rosenbrock, State Space and Multivariable Theory, John-Wiley, New York, 1970.

[13] M. Saif and Y. Guan, "A new approach to robust fault detection and identification," IEEE Trans. Aerospace and Electronic Systems, vol. 29, pp. 685-695, 1993.

[14] C. P. Tan and C. Edwards, "Sliding mode observers for detection and reconstruction of sensor faults," Automatica, vol. 38, pp. 1815-1821, 2002.

[15] C. P. Tan and C. Edwards, "Sliding mode observers for robust detection and reconstruction of actuator and sensor faults," International Journal of Robust and Nonlinear Control, vol. 13, pp. 443-463, 2003.

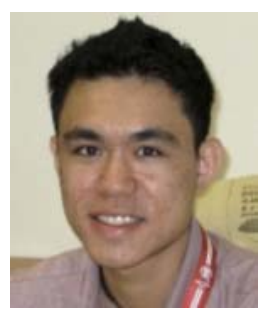

Kok Yew Ng received his B.Eng (Hons) and Ph.D. in Control System from Monash University in 2005 and 2009, respectively. He was then subsequently appointed as Lecturer at the School of Engineering, Monash University Sunway Campus (Malaysia). His research interests include disturbance decoupling, fault reconstruction and fault tolerant.

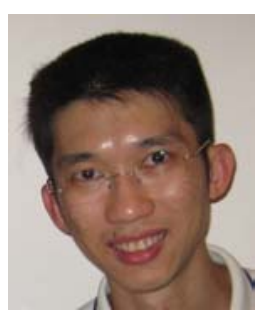

Chee Pin Tan received his B.Eng. (1st class honours) in 1998, and a Ph.D. in 2002 from Leicester University, U.K. He was then appointed as Lecturer in 2002 at the School of Engineering, Monash University Sunway campus (Malaysia) and subsequently promoted to Senior Lecturer in 2008. His main research interests include robust fault reconstruction and sliding mode observers. He has published over 50 internationally peer-reviewed research articles. In addition, he received the Pro-Vice-Chancellor (PVC) Award for Distinguished Teaching 2006 and the PVC Award for Excellence by Early Career Researchers 2007. 


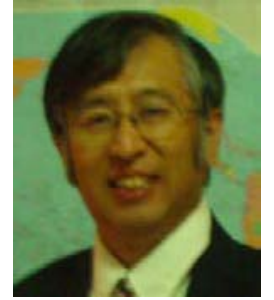

Zhihong Man received his B.Eng degree from Shanghai Jiaotong University, China, in 1982, an M.Sc degree from Chinese Academy of Sciences in 1987, and a Ph.D. degree from the University of Melbourne, Australia, in 1994, respectively. From 1994 to 1996 , he was the Lecturer in the Department of Computer and Communication Engineering, Edith Cowan University, Perth, Australia. From 1996 to 2001, he was the Lecturer and then Senior Lecturer in the School of Engineering, The University of Tasmania, Australia. In 2001, he was the Visiting Senior Fellow in the School of Computer Engineering, Nanyang Technological University (NTU), Singapore. From 2002 to 2007, he was the Associate Professor of Computer Engineering at NTU. From 2007 to 2008, he was with Monash University Sunway Campus (Malaysia) and served as the Professor of Electrical and Computer Systems Engineering, the Chair of the Research Committee of the School of Engineering, and the Chair of the Campus Research Committee. Since 2009, he has been with the Faculty of Engineering and Industrial Sciences, Swinburne University of Technology, Melbourne, Australia, where he is the Professor of Engineering and the Head of Robotics and Mechatronics. Professor Man's research interests are in nonlinear control, signal processing, robotics, neural networks, fuzzy systems, engineering optimization and wireless communication. He has published more than 150 papers in refereed international journals and refereed international conferences proceedings in these areas. Since 1994, Professor Man has been involved in more than 30 international conferences in control, robotics, signal processing, neural networks and industrial electronics as Program Committee Chair, Track Chair, Session Chair, and the International Advisory Committee and Technical Committees member, including the Chair of the Program Committee of the third IEEE International Conference on Industrial Electronics and Applications (ICIEA 2008) and the co-Chair of the Program Committee of the Fourth IEEE International Conference on Industrial Electronics and Applications (ICIEA 2009). In addition, Professor Man received the NTU Best Teacher Award in 2004, and the Most Popular Lecturer in the School of Computer Engineering at NTU from 2002 to 2007.

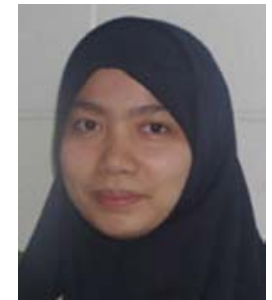

Rini Akmeliawati was born in Jakarta, Indonesia, in 1975. She obtained the Bachelor of Engineering (Honours) in Electrical Engineering from Royal Melbourne Institute of Technology (RMIT) University, Australia in 1997, and her Ph.D. in Electrical and Electronic Engineering from the University of Melbourne, Australia in 2002. She is currently an Associate Professor of the Department of Mechatronics Engineering at the International Islamic University Malaysia. Previously, she was a lecturer at RMIT University (1997-2001) and Monash University Sunway Campus (2001-2004). Her main research interests include nonlinear control systems theory and applications, intelligent systems and image processing. Rini Akmeliawati is a Senior Member of IEEE. She is the Chair of IEEE Instrumentation and Measurement Society - Malaysia Chapter 2007-2009. 